\title{
Molecular characterization of Dg3, a cDNA that encodes a novel lipid transfer protein in Brassica napus
}

\begin{abstract}
In this study, we have analysed the sequence of $\mathrm{Dg} 3$ clone using bioinformatic tools, determined copy number of this transcript in the genome of $\mathrm{B}$. napus and expression levels at various tissues/organ. The cDNA contained 307-base pair open reading frame encoding 102 amino acid residues, 60-base pair 5'-untranslated region and 127-base pair 3 -untranslated region. The predicted mature protein has a molecular weight of $9.2 \mathrm{kDa}$ and is acidic, with a predicted isoelectric point $(\mathrm{pI})$ of 6.2. The Dg3 sequence has all the conserved structural characteristics of plant LTPs and showed highest homology to LTPs from other plant species. The transcripts of Dg3 were detected in all tested tissues but highest expression was in siliques and in vitro embryogenic cultures. Possible roles of Dg3 during somatic embryogenesis and normal plant development are discussed.
\end{abstract}

Keyword: Lipid transfer protein; cDNA; B.napus; Transcript; Expression analysis 